\title{
Visual transformations underlying apparent movement
}

\author{
JOYCE E. FARRELL \\ New York University, New York, New York
}

\begin{abstract}
Apparent movement of a rigid object is seen when two different views of the same object are separated by relatively long distances and times. In a series of experiments, the minimum stimulus-onset asynchrony (SOA) required for the apparent form-preserving movement of an object was recorded. In Experiment 1, an object was alternately displayed in two positions obtained by displacing and rotating the object about the center of the display screen. In Experiment 2, objects varying in size were presented in alternate positions, again differing by both location and orientation. In Experiment 3, stimulus location and orientation were varied orthogonally. In all three experiments, the minimum SOA was predicted by a linear combination of the distance separating the two locations of the object and the angle separating the two orientations of the object. The results support the hypothesis that the long-range apparent movement of a rigid object may be constructed by internal processes corresponding to rotation and translation of the object.
\end{abstract}

When successive views of an object are presented in appropriate spatial and temporal positions, observers report an experience of movement that is similar to that generated when the object moves smoothly from the first position to the second. The movement is called "apparent" to emphasize that there is no physical movement, but merely an impression of continuous movement. When successive stimuli are separated by relatively small distances and times, such as in successive frames of a film, the perception of movement may be indistinguishable from real continuous movement. Observers also report the sensation of movement, however, when successive stimuli are separated by large distances and times, and the percept is quite distinguishable from real movement.

It has been suggested that apparent movement over short distances is directly sensed by motion-detecting mechanisms, whereas apparent movement over long distances is indirectly inferred from higher level interpretive processes (Braddick, 1974). Support for this notion comes from the finding that apparent

This work was done in partial fulfillment of the doctoral dissertation requirement in psychology at Stanford University. The work was supported by a National Institute of Mental Health training grant to Joyce E. Farrell and National Science Foundation Research Grant BNS-75-02806 to Roger N. Shepard. I thank Geoffrey Loftus, Roger Remington, Brian Wandell, and Dan Weintraub for their comments on an earlier draft of the paper. I thank Ewart Thomas, Brian Wandell, and A. Beau Watson for many helpful discussions of the work. And I thank Claus Bundesen, Axel Larsen, and Roger Shepard for their advice and encouragement during all phases of the project. My present mailing address is: Department of Psychology, New York University, New York, New York 10003. movement over short distances is determined by the retinal separation of successive stimuli, whereas apparent movement over long distances is determined by the apparent separation of stimuli in threedimensional space (Attneave \& Block, 1973; Baker \& Braddick, 1982; Larsen, Farrell, \& Bundesen, Note 1; cf. Lappin, Doner, \& Kottas, 1980).

A theory of apparent movement over long distances will have to account for several important findings: First, the minimum temporal interval separating the onset of successive stimuli-the stimulusonset asynchrony (cf. Kahneman, 1967; Kahneman \& Wolman, 1970; Sgro, 1963) required for the apparent translation of a rigid object-is a linearly increasing function of the apparent separation of successive views of the object in three-dimensional space (Attneave \& Block, 1973; Corbin, 1942; Larsen, Farrell, \& Bundesen, Note 1). Second, the minimum stimulus-onset asynchrony (SOA) required for the apparent rotation of a rigid object is linearly increasing function of the angle separating successive views of the object, and the linear function is nearly identical for perceived rotations in depth and in the picture plane (Shepard \& Judd, 1976).

Long-range apparent movement is presumably generated by a central process dependent on prior computation of the positions of the stimuli in threedimensional space. Here I adopt the working hypothesis that, when presented with two successive views of an object that are widely separated in space and time, the perceptual system attempts to interpolate a path connecting the successive stimuli by identifying corresponding parts in the two successive views (Ullman, 1979) and then connecting the corresponding parts by an internal transformation that passes through a se- 
quence of representations similar to those that would be expected if the object were actually presented in intermediate positions (Robins \& Shepard, 1977). The theoretical interpretation of the slope and intercept of the linear relation between perceived separation and the minimum SOA required for the perception of movement (Attneave \& Block, 1973; Corbin, 1942; Shepard \& Judd, 1976; Larsen, Farrell, \& Bundesen, Note 1) is that the intercept measures the time taken to identify two successive stimuli as representing the same object at different times and that the slope measures the maximum speed of visually interpolating a path connecting the successive stimuli.

In these experiments, I examine the nature of the long-range interpolative process. The experiments of Farrell, Larsen, and Bundesen (1983) suggest that the interpolation of apparent translational movement is constrained by a maximum linear velocity of the object in apparent 3-D space and the interpolation of apparent rotational movement is constrained by a maximum angular velocity of the object. Because the minimum SOA for apparent rotation varies linearly with the angle of rotation, such that the slope of the function is constant regardless of the size of the object, Farrell et al. concluded that the interpolation of apparent rotation is constrained by an upper bound on angular velocity of the object as a whole, rather than by a limit on the linear velocities of the individual parts.

Bundesen, Larsen, and Farrell (Note 2) also provided evidence that apparent translational and apparent rotational movement are mediated by separate object-based interpolative mechanisms. In their experiment, disparities of size produced translatory movement in depth, and disparities in both orientation and size produced impressions of screwlike or helical movement in depth. The SOA thresholds for apparent form-preserving movement could be predicted on the assumption that interpolation times for apparent translation in depth and apparent rotation combined additively.

Here I directly test the hypothesis that the interpolative process may be decomposed into two submechanisms. The first submechanism is responsible for computation of the rectilinear displacement of the object, while a second, independent mechanism computes the angular rotation of the object. This hypothesis may be contrasted with the alternative hypothesis that two visual shapes can be brought into correspondence by a rotation in the image plane performed by a single mechanism. Rectilinear motion is the special case, then, of the action of this rotational mechanism when the point of rotation is at infinity.

To contrast these hypotheses, I alternately presented two visual shapes differing in location and orientation and measured the minimum SOA that resulted in the perception of a rigid object moving continuously within the plane. If the perceptual sys- tem constructs but a single rotation, the minimum SOA should be a linearly increasing function of the angular difference in orientation alone. If the perceptual system constructs a translation and a rotation, the minimum SOA should be determined by angular difference in orientation as well as the linear separation.

\section{EXPERIMENT 1}

\section{Method}

Subjects. Eight students at Stanford University participated in this experiment. All participants had normal or corrected-tonormal vision. The participants had no previous exposure to experiments in visual perception and were not aware of the purpose of the study.

Stimuli. The stimulus, shown in Figure 1a, was alternately displayed in different positions on a computer-driven cathode ray tube (a Megatek calligraphic display system equipped with a P4 phosphor) that was refreshed every $11 \mathrm{msec}$. The intensity of the stimulus was set such that a 1-deg vector was equivalent to $.2 \mathrm{fL}$. The subject viewed the display binocularly from a distance of $1 \mathrm{~m}$. At this viewing distance, the stimulus subtended a visual angle of approximately 1.72 deg. Stimuli were viewed binocularly with free fixation.

During a trial, the stimulus was alternately displayed in different screen locations obtained by displacement of the stimulus from the center of the screen and by rotations about the screen center, as shown in Figure 1a. The stimulus was presented in 12 combinations of orientations. If $u$ is one of the angular orientations in which the stimulus was shown and $u+V$ is the other orientation, then the 12 combinations are given by: $u$ deg, $u+V(\bmod 360)$ deg (for $V=30,60,90$, and 120 deg and $u=0,30, \ldots, 330$ deg). For each pair of stimulus orientations, displacement from the screen center was $0,1.5,3.0$, or $4.5 \mathrm{~cm}$. There were a total of $192 \mathrm{stim}$ ulus pairs, which were presented in a random order.

Procedure. On each trial, the two stimuli in a pair were displayed in sequential alternation with a blank interstimulus interval of $50 \mathrm{msec}$, as illustrated in Figure 1b. The interstimulus interval insured that the phosphor image trace of the display had decayed before the presentation of the alternate image. The stimulus duration was initialized at $1,000 \mathrm{msec}$, thus setting the stimulus-onset asynchrony (SOA) at 1,050 msec. At this rate of alternation, the subjects reported seeing a single object moving back and forth along a path within the picture plane while preserving its shape. The subjects tapped a right-hand key, which decreased the stimulus duration (or, equivalently, the SOA) by $1 / 11$ th, until apparent form-preserving movement was replaced by partial movement and/or flicker. [The subjects typically reported that at the transition point (between apparent form-preserving movement and partial movement) the straight line of the pattern appeared to move while the curved sector elements simply flickered.] The resulting limiting value of SOA, referred to as SOA1, was recorded when the subjects pushed a center key. The subjects then tapped a left-hand key, which increased the SOA by $1 / 10$ th, until apparent form-preserving movement reappeared. Finally, they pressed a center key to record this limiting value of SOA, referred to as SOA2, and to terminate the trial.

The entire experiment took four 1-h sessions. In each session, the subjects completed 48 trials.

\section{Results and Discussion}

SOA2 is proportional to SOA1 $(r=.999)$. The mean of these two threshold measures, averaged across eight subjects, will be referred to as the "minimum SOA."

Two hypotheses will be considered. The first hy- 
(a)
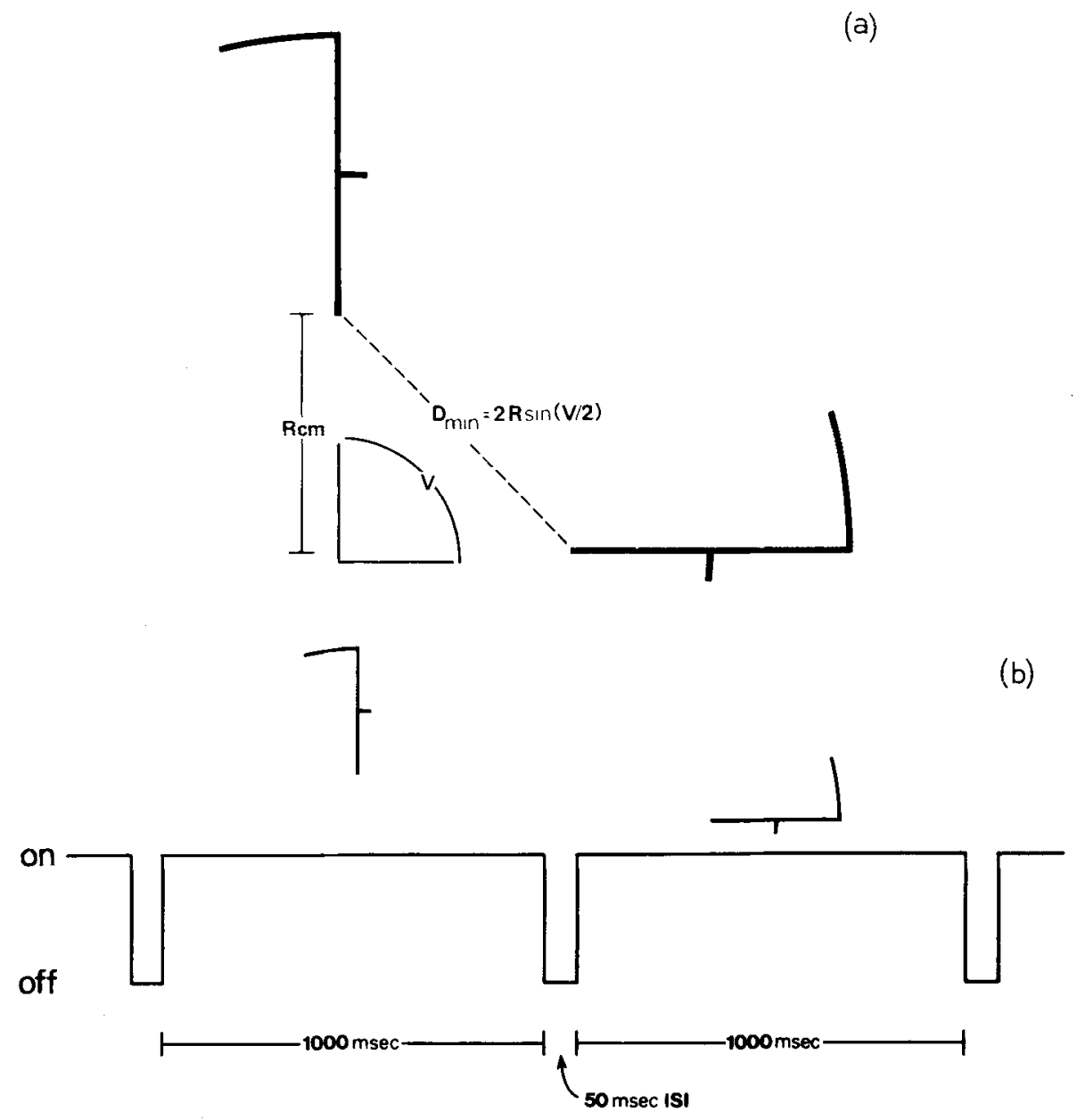

Figure 1. (a) Stimulus pattem shown in two orlentations differing by $V$ deg and displaced from from the screen center by $\mathbf{R ~ c m}$. D is the linear distance separuting nearest corresponding parts of the object. (b) At the beginning of each trial, the stimulus pattern was presented for 1,000 meec in alternate positions separated by a blank interstimulus interval (ISI) of 50 msec. The stimulus-onset asynchrony was adjusted by varying stimulus duration.

pothesis, hereafter referred to as the single rotation hypothesis, is that the interpolation of apparent movement between alternate views of an object differing in location and orientation is mediated by a single internal transformation constrained by an upper bound on angular velocity. This hypothesis predicts the minimum SOA to increase linearly with the angular difference in orientation, that is,

$$
\text { minimum SOA }=a+b V,
$$

where the angle of rotation, $\mathrm{V}$, is computed about a point that may be at some distance from the object. The intercept, a, measures the time taken to establish a correspondence between the two stimulus presentations or, in other words, to identify the two presentations as representing the same object at different times. The slope constant, $b$, is determined by the velocity constraint on the interpolation of a path of rotation.
The second hypothesis, hereafter referred to as the rotation plus translation hypothesis, states that the perceptual system interpolates a path of movement by two separate internal processes corresponding to rotation of the object through the minimum angle and translation of the object through the minimum linear distance separating the two positions. Moreover, the perceptual system combines rotations and translations such that transformation times combine additively (Bundesen, Larsen, \& Farrell, Note 2). An additive structure of transformation times would be consistent with the idea that the interpolation processes corresponding to rotations and translations of the object are separate. Briefly,

$$
\text { minimum } \mathrm{SOA}=\mathrm{a}+\mathrm{bV}+\mathrm{cD},
$$

where $\mathrm{V}$ represents the minimum angle separating object orientations, and $\mathrm{D}$ is the minimum linear distance separating corresponding object parts. 
Again, the intercept constant, $a$, is the time required to identify successive stimuli as representing the same object and the slope constants, $\mathrm{b}$ and $\mathrm{c}$, are determined by velocity limits on interpolation processes corresponding to transformations of rotation and translation, respectively.

An implicit assumption is that the internal rotation transformation interpolates motion across the minimum angle separating object orientation, $\mathrm{V}$, and the internal translation transformation interpolates motion across the minimum linear distance separating object location, $D$. This assumption reflects the observation that, in instances of pure rotation, observers report that the object rotates through the minimum angle, $\mathrm{V}$, and not the larger complementary angle, 360-V (Farrell \& Shepard, 1981; Shepard \& Judd, 1976; Wertheimer, 1912). Similarly, in cases of pure translation, observers report that the object traverses the shortest of possible paths (Wertheimer, 1912). The assumption of minimum distance is not critical, however, since any linear transformation of $V$ or $D$ will not affect the regression analysis.

Figure 2a shows the "minimum SOA," plotted as a function of the angular difference between orientations of alternate stimuli (V) with displacement of the shape from the screen center (R) as the parameter. Recall that the single rotation hypothesis, expressed in Equation 1, predicts the minimum SOA to increase linearly with the angle $V$. I tested the singlerotation hypothesis by fitting linear functions relat- ing minimum SOA and angular difference to the data for each displacement of the object from the screen center, with the constraint that the slopes of the lines be the same. The linear functions, shown in Figure $2 a$, were fitted to the data by an iterative method (see Chandler, Note 3). These theoretical functions account for approximately $88 \%$ of the variance in mean minimum SOAs. The root mean square error between predicted and obtained results is $24 \mathrm{msec}$. Furthermore, a two-way analysis of variance with repeated measures indicates a significant interaction between angular difference and displacement from the screen center $[F(9,63)=6.38$, $p<$ $.001]$. Equation 1 cannot account for this interaction.

Figure $2 \mathrm{~b}$ shows the minimum SOA plotted as a function of the minimum distance between the stimulus and the screen center, $\mathbf{R}$, with the angular difference in stimulus orientation, $V$, as the parameter. The minimum linear distance separating nearest corresponding parts, $D$, is given by $2 R \sin (V / 2)$ (see Figure 1). Recall that the rotation plus translation hypothesis postulates two separate interpolation processes corresponding to rotation through the minimum angle and translation through the minimum distance. This hypothesis predicts the minimum SOA by a linear combination of the angular difference in stimulus orientation, $V$, and the linear separation of stimulus position, $2 R \sin (V / 2)$ (see Equation 2). If the hypothesis is correct, the intercepts of functions relating the minimum SOA to $\mathrm{R}$ in

(a)

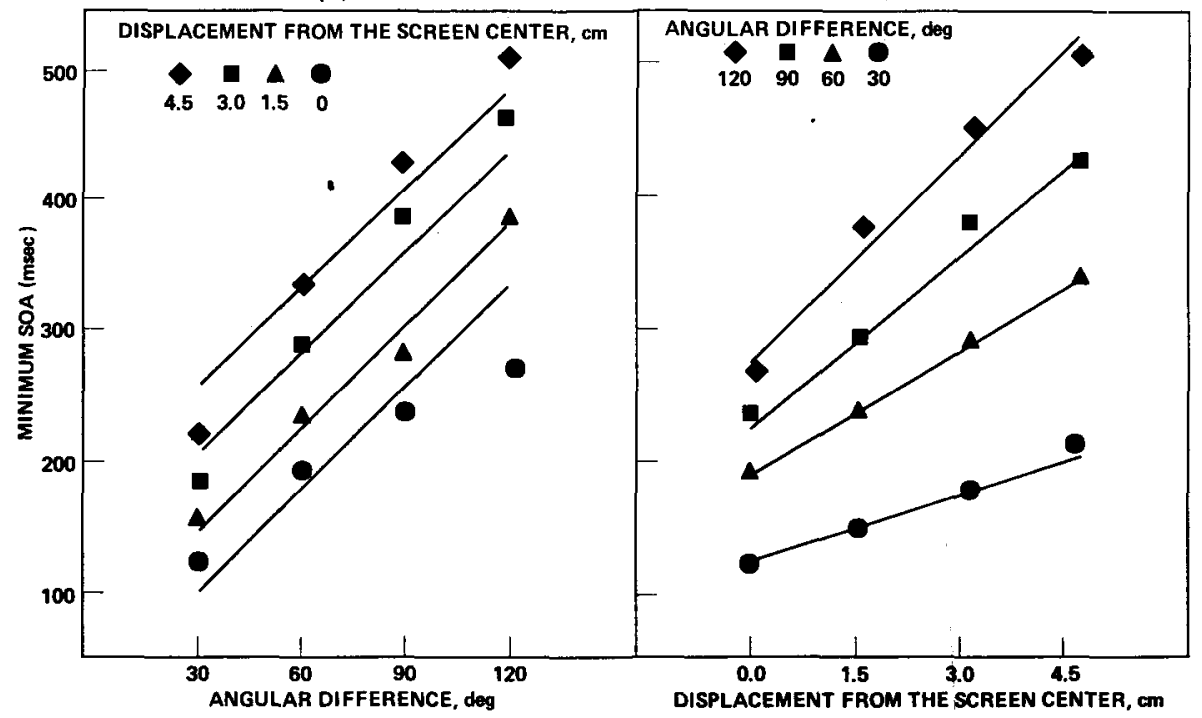

Figare 2. (a) Group means of minimum SOA as a function of the angular difference in orientation between the stimull with stimulas displacement from the sereen center as the parnmeter. Straight lines cuperimposed on the data vary in Intercept but not slope. (b) Gronp means of minimum SOA as a function of stimulus displacement from the screen center with angular difference in ortentation between the stimuli $(V)$ as the parameter. Straight lines vary in intercept, and slopes are proportional to $\sin (\mathrm{V} / 2)$. 
Figure $2 \mathrm{~b}$ should vary with $\mathrm{V}$ and the slopes should vary with $\sin (\mathrm{V} / 2)$. In order to contrast the rotation plus translation hypothesis with the single rotation hypothesis, I again fit four lines, one for each angle of rotation, but with the constraint that the slopes of the lines be proportional to $\sin (\mathrm{V} / 2)$. The best-fitting functions, obtained by iterative curve-fitting, are shown superimposed on the data in Figure $2 \mathrm{~b}$. These theoretical functions account for approximately $99 \%$ of the variance in mean minimum SOAs, and the root mean square error between predicted and obtained results is only $10 \mathrm{msec}$.

The results-namely, that the minimum SOA for apparent rigid movement was proportional to the distance separating nearest corresponding points and the minimum angle separating alternate orientations of the object-support the hypothesis that the path of apparent rigid movement was constructed by separate internal processes (corresponding to rotation and translation transformations of the object) which are constrained by angular and linear velocity, respectively. However, an alternative interpretation of the results is that the path was constructed by a single process constrained by an upper bound on linear velocity of object parts (cf. Farrell, Larsen, \& Bundesen, 1982). I examine this hypothesis in the next experiment by holding the maximum separation of object parts constant while varying the distance separating nearest corresponding parts. If path interpolation is mediated by a single process constrained by an upper bound on linear velocity of the moving parts of the object, then the minimum SOA for apparent movement should be proportional to the maximum length of the trajectory of any moving part. But if path interpolation is mediated by two separate processes constrained by angular and linear velocity, respectively, then the minimum SOA for apparent movement should be proportional to the angle of rotation and the path of translation.

\section{EXPERIMENT 2}

\section{Method}

Subjects. The same eight subjects who participated in Experiment 1 were also the subjects in this second experiment.

Stimull. Four versions of the original prototype stimulus were constructed by varying the length of the line segment, $L$, where $\mathrm{L}=1.5,3.0,4.5$, or $6.0 \mathrm{~cm}$ (see Figure 3). From the viewing distance of $1 \mathrm{~m}$ these stimulus patterns subtended a visual angle of $.86,1.72,2.58$, or $3.43 \mathrm{deg}$, respectively.

As in the previous experiment, each stimulus pattern was alternately displayed in different screen locations obtained by rotations and displacements with respect to the center of the screen. Again, each stimulus pattern was presented in 12 combinations of orientations, given by: $u$ deg, $u+V(\bmod 360) \operatorname{deg}($ for $V=30,60$, 90 , or $120 \mathrm{deg}$ and $u=0,30, \ldots, 330 \mathrm{deg}$ ). As shown in Figure 3, displacement from the screen center corresponds to $\mathbf{M}$, where $\mathbf{M}=$ $6.0 \mathrm{~cm}-\mathrm{L}$, and the radius of rotation, $R$, was the same for all stimuli $(R=6.0 \mathrm{~cm})$.

The total set of 192 stimulus pairs was presented in a random

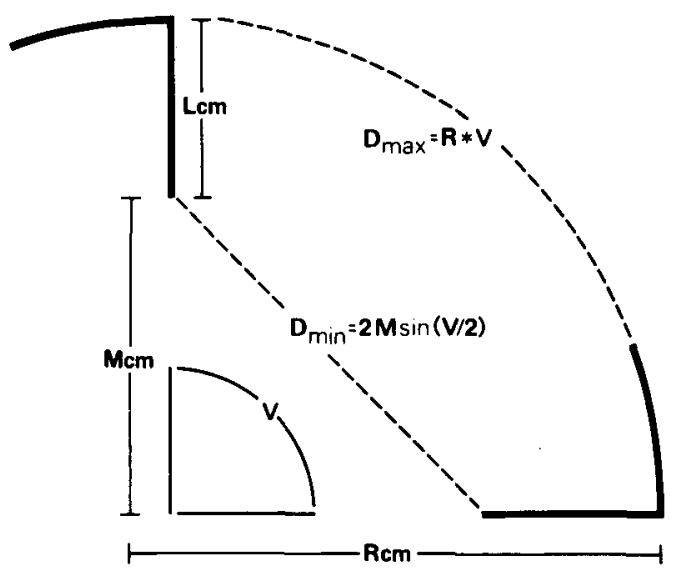

Figure 3. One of four stimulus patterns shown in two orientations differing by $V$ deg and displaced from the screen center by $M \mathrm{~cm} . \mathrm{D}_{\mathrm{mia}}$ is the linear distance separating nearest corresponding parts of the object. $D_{\max }$ is the length of the trajectory of a point on the object moving farthest (and therefore fastest) from center of rotation. Maximum displacement from the screen center, $\mathbf{R}$, was constant.

order, divided into blocks of 48 , and viewed on 4 consecutive days.

All other aspects of the stimulus conditions remained identical to Experiment 1.

Procedure. As in previous experiments, the two stimuli in a pair were presented in sequential alternation with a constant interstimulus interval of 50 msec. The stimulus-onset asynchrony (SOA) was initialized at $1,050 \mathrm{msec}$. Observers decreased the SOA until the illusion of form-preserving movement disappeared. They then increased the SOA until they could once again perceive continuous form-preserving movement.

\section{Results and Discussion}

As in the previous experiment, SOA2 is proportional to SOA1 $(r=.999)$. The mean of these two threshold measures, averaged across eight subjects, is referred to as the minimum SOA for apparent movement.

Again, I will contrast two hypotheses. The first hypothesis is that the interpolation of a path between two views of an object which differ in location and orientation is constructed by a single process constrained by the maximum linear velocity. This hypothesis, hereafter referred to as the single translation, hypothesis, predicts

$$
\text { minimum SOA }=a+b D_{\max }
$$

where $a$ is the time to determine a correspondence between two views of an object and $b$ is the velocity constraint on the interpolation of a path connecting the two views. $D_{\max }$ is the length of the trajectory of a point on the object moving farthest (and therefore fastest) from center of rotation. $D_{\max }$ is estimated by the product of the radius of rotation, $R$, and the minimum angle of rotation, $V$ (see Figure 3 ). Equation 3 implies, then, that the minimum SOA for 
apparent movement of a rigid object is determined by the linear velocity of the fastest moving object part. Since the radius of rotation was held constant, the minimum SOA should be proportional to the angle separating alternate orientations of the object.

The second hypothesis is that the perceptual system interpolates a path between two views of an object differing in location and orientation by separate processes constrained by linear and angular velocity of the object as a whole. This hypothesis, referred to as the rotation plus translation hypothesis, predicts the minimum SOA to be proportional to the distance separating nearest corresponding parts of the object and the minimum angle separating alternate orientations of the object (see Equation 2).

Figure $4 a$ shows the minimum SOA plotted as a function of the angular difference between alternate orientations of the object. I tested the single translation hypothesis by fitting linear functions relating minimum SOA and angular difference to the data for each stimulus pattern with the constraint that the slopes of the lines be the same, as predicted by Equation 3. The theoretical functions, shown in Figure $4 a$, account for approximately $88 \%$ of the variance in mean minimum SOA. The data depart from the predictions by a root mean square error of $25 \mathrm{msec}$. The interaction between angular difference and displacement of the stimulus from the screen center is significant at the .01 level $[F(9,63)=11.39]$. The single translation hypothesis, expressed in Equation 3, fails to account for this interaction.

Figure $4 \mathrm{~b}$ shows the minimum SOA plotted as a function of the extent of displacement of the stimulus from the screen center, $M$. The minimum distance separating corresponding object parts is given by $2 \mathrm{M}[\sin (\mathrm{V} / 2)]$, where $\mathrm{V}=$ the minimum angle separating object orientations. The rotation plus translation hypothesis - that path interpolation was mediated by separate internal processes constrained by linear and angular velocity of the object as a whole-was tested by fitting four lines to the data, one for each angle of rotation, with the constraint that the slopes of the lines be proportional to $\sin (\mathrm{V} / 2)$. These theoretical functions account for $97 \%$ of the variance. The obtained results depart from the prediction with a root mean square error of $9.8 \mathrm{msec}$.

The results of Experiments 2 and 3 are explained on the assumption that the interpolation or transformation times for internal translations and rotations combine additively. That is,

$$
\text { minimum SOA }=a+b V+c D,
$$

where a estimates a delay period preceding the transformational processes and $\mathbf{b}$ and $\mathbf{c}$ are determined by the rate-limiting constraints on the internal transformations of rotation and translation, respectively. Estimates of the hypothetical parameters (see Equation 2 above) were obtained by an iterative search for values of $\mathbf{a}, \mathbf{b}$, and $c$ that minimized the root mean square error between the predicted and the obtained results. A confidence interval around each estimate was determined by the constant that, when added to or subtracted from the parameter estimate, would increase the root mean square error by a factor of two. An analysis of the results obtained in Experi-

(a)

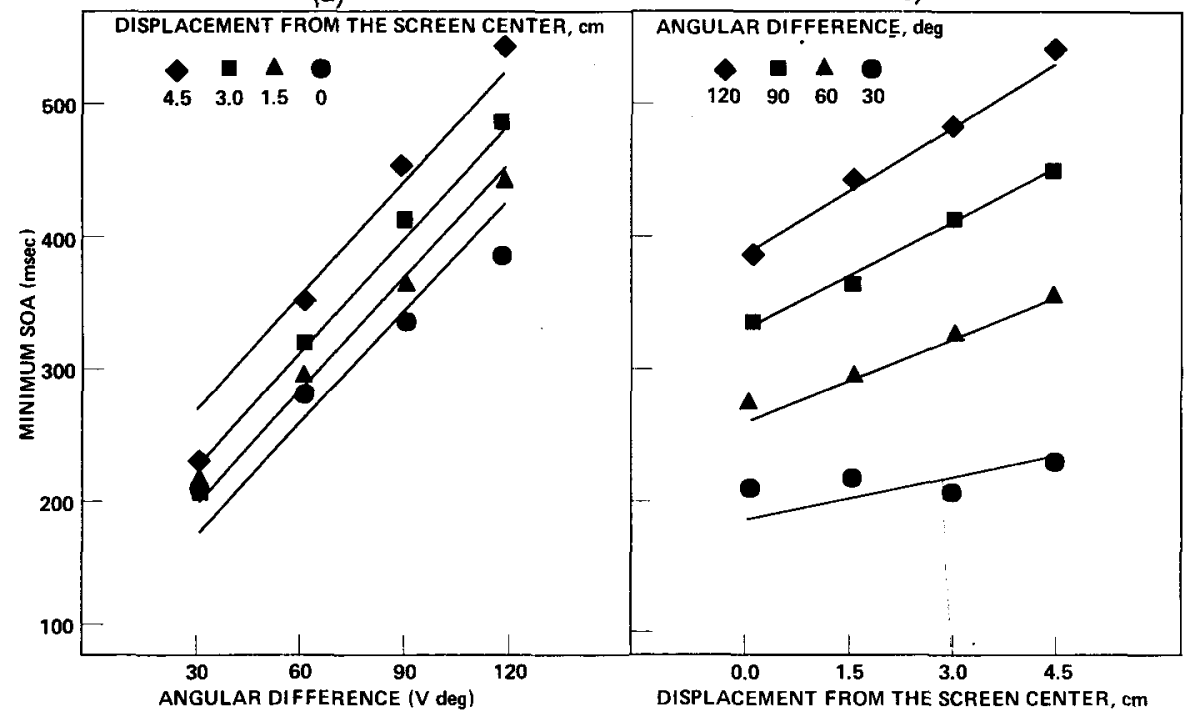

Figure 4. (a) Group means of minimum SOA as a function of the angular difference in stimulus orientation with displacement from the screen center, $M$, as the parameter. (b) Group means of minimum SOA as a function of stimulus displacement from the screen center with angular difference in orientation between the stimuli $(V)$ as the parameter. Straight lines vary in intercept, and slopes are proportional to $\sin (\mathrm{V} / 2)$. 
ment 1 yields $a=86 \mathrm{msec}-20, b=1.59 \mathrm{msec} / \mathrm{deg}-$ .25 , and $c=32.41 \mathrm{msec} / \mathrm{cm}-6.0$. Analysis of the data obtained in Experiment 2 yields $a=129-18$, b $=2.18 \mathrm{msec} / \mathrm{deg}-.20$, and $\mathrm{c}=19.52 \mathrm{msec} / \mathrm{cm}$ -5.5. The estimate of $c$ obtained from the data of Experiment 2 may be less reliable due to the residual departures between the hypothetical and empirical outcomes for stimuli differing in orientation by $30 \mathrm{deg}$ (see Figure 4b). The remaining differences in parameter estimates of Experiment 1 and Experiment 2 may be due to differences in the stimulus conditions of the two experiments. For example, in Experiment 1, stimulus size was constant but retinal location varied. In Experiment 2, retinal location was constant but stimulus size varied.

In order to obtain a third independent estimate of the hypothetical parameters and to further test the additivity implied in Equation 2, I performed the following experiment in which stimulus location and orientation were varied in an explicitly orthogonal way.

\section{EXPERIMENT 3}

\section{Method}

The method was identical to that of Experiments 1 and 2, except as noted.

Stimuli. The prototype shape of the preceding experiments was again used here. In a given trial, the stimulus pattern was alternately presented in 2 of 8 screen locations and in 2 of 12 orientations differing from the standard upright by $0,30, \ldots$, or $330 \mathrm{deg}$ of positive rotation in the picture plane. The difference in angular orientation between the two members of a pair, $V$, was 0,30 , 60 , or $90 \mathrm{deg}$. The two members of a pair were symmetrically displaced in opposite directions along a horizontal axis passing through the center of the screen. The minimum linear distance separating stimulus pair, D, was $0,1.5,3.0$, or $4.5 \mathrm{~cm}$. Figure 5 shows examples of the stimulus combinations. There were a total of 180 stimulus pairs which were randomly arranged and presented in four blocks of $\mathbf{4 5}$ trials.

\begin{tabular}{|c|c|c|c|}
\hline & 77 & 77 & 77 \\
\hline 72 & 77 & 77 & 77 \\
\hline$\sqrt{2}$ & +7 & $\neq 7$ & $\uparrow$ \\
\hline$x$ & $\uparrow 7$ & $\approx 7$ & 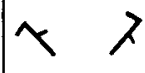 \\
\hline
\end{tabular}

Figure 5. Examples of stimulus pair combinations created by orthogonal variation of two parameters: the distance separating stimulus location and the angle separating stimulus orientation.
Subjects. Eleven individuals participated in this experiment, but only eight were retained as subjects. Two individuals were excluded from the experiment because they reported that they did not understand the criteria of rigid vs. nonrigid movement and gave judgments that were excessively variable. The data from one individual were not considered because she gave threshold values that were twice as large as those obtained from all other subjects, although her data were similar in all other respects. All participants had normal or corrected-to-normal vision, had had no prior experience as subjects in perception experiments, and were unaware of the purpose of the study.

\section{Results and Discussion}

Figure 6 shows the minimum SOA for the eight retained subjects plotted as a function of the angular difference in orientation of stimuli (V) with the minimum linear distance separating stimuli (D) as the parameter. The straight lines superimposed on the data in Figure 6 are the predictions given by the additivity hypothesis-namely,

$$
\text { minimum SOA }=a+b V+c D .
$$

Again, the parameter " $a$ " represents the time to begin visual transformations. The variables $b$ and c represent rate-limiting constraints on rotation and translation transformations, respectively. Using an iterative curve-fitting procedure, I estimated $a=$ $75 \mathrm{msec}-14, \mathrm{~b}=.8 \mathrm{msec} / \mathrm{deg}-.24$, and $\mathrm{c}=28.76$ $\mathrm{msec} / \mathrm{cm}-5.5$. The additivity hypothesis accounts for approximately $93 \%$ of the variance. The root mean square error between the predicted and observed results is $8 \mathrm{msec}$.

The minimum SOA for apparent rigid movement between two views of an object can be predicted in terms of a function relating minimum SOA to the linear distance separating the location of the object in the two views and to the angle separating the orientation of the object in the two views. On the average, the results depart from these predictions by only $8 \mathrm{msec}$. The results are explained on the assumption that the subjective interpolation of a path connecting two views of a rigid object is constructed by two separate processes corresponding to internal transformations of rotation and translation of the object. The hypothetical image transformations are constrained by separate velocity limits on movement of the object as a whole: Perceptual rotation transformations are limited by a maximum angular velocity, and translation transformations are limited by a maximum linear velocity.

The additive structure of transformation times may reflect the serial action of two separate processes or the capacity limitations of two separate processes when they occur in parallel. Bundesen, Larsen, and Farrell (Note 2) suggested that apparent rotations and apparent translations in depth were mediated by sequential alternation of two separate processes such that small steps of rotation alternated with small steps of translation. When the steps are very small, the serial action of two separate processes will be in- 


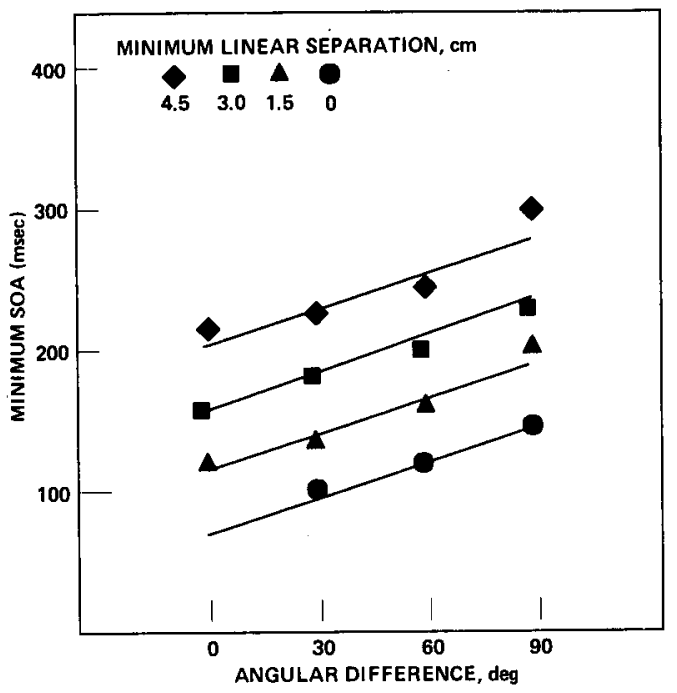

Figure 6. Group means of minimum SOA as a function of the angular difference in orientation between stimull with minimum linear distance between stimuli as the parameter.

distinguishable from the parallel action of two capacity-limited processes. Thus, the order of transformation is unresolved. However, the additivity of transformation times suggests that the internal process mediating long-range apparent movements of rigid objects can be separated into two objectbased interpolative mechanisms corresponding to rotations and translations of the object.

\section{SUMMARY}

I report a series of experiments that investigate the apparent interpolation of a path connecting two views of an object differing in location and orientation. The results support the hypothesis that subjective interpolation of movement over long distances is constructed by two separate processes: a process constrained by a limiting velocity of rectilinear apparent translation of the object and a process constrained by a limiting velocity of angular apparent rotation of the object. In Experiments 1 and 2, I rule out alternative hypotheses that path interpolation is constructed by a single process corresponding to rotation of the image plane (constrained by an upper bound on angular velocity) or translation of object parts (constrained by an upper bound on linear velocity). In Experiment 3, I show that breakdowns in the apparent movement of a rigid object can be predicted by the joint action of two separate interpolation processes constrained by the angular velocity of object rotation and the linear velocity of object translation. The results support the conjecture that long-range apparent movement is constructed by two independent processes for interpolation of rotation and translation.

\section{REFERENCE NOTES}

1. Larsen, A., Farrell, J. E., \& Bundesen, C. Visual apparent movement: Evidence for separate short-and long-range processes. Manuscript submitted for publication, 1983.

2. Bundesen, C., Larsen, A., \& Farrell, J. E. Apparent visual transformations of size and orientation. Manuscript submitted for publication, 1983.

3. Chandler, J. P. STEPIT (Quantum Chemistry Program Exchange). Bloomington: Department of Chemistry, Indiana University, 1965.

\section{REFERENCES}

Atrneave, F., \& Block, G. Apparent movement in tridimensional space. Perception \& Psychophysics, 1973, 13, 301-307.

Braddick, O. A short-range process in apparent motion. Vision Research, 1974, 14, 519-528.

Consin, H. H. The perception of grouping and apparent movement in visual depth. Archives of Psychology, 1942, No. 273, $1-50$.

Farrell, J. E., Largen, A., \& Bunde8en, C. Velocity constraints on apparent rotational movement. Perception, 1983, in press.

Farrell, J. E., \& Shepard, R. N. Shape, orientation, and apparent rotational motion. Journal of Experimental Psychology: Human Perception and Performance, 1981, 7, 477-486.

Kahneman, D. An onset-onset law for one case of apparent motion and metacontrast. Perception \& Psychophysics, 1967, 2, 577-584.

Kahneman, D., \& Wolman, R. E. Stroboscopic motion: Effects of duration and interval. Perception \& Psychophysics, 1970, 8, 161-164.

Lappin, J. S., Doner, J. F., \& Kotras, B. L. Minimal conditions for the visual detection of structure and motion in three dimensions. Science, 1980, $209,717-719$.

Robins, C., \& SHePARD, R. N. Spatio-temporal probing of apparent rotational movement. Perception \& Psychophysics, 1977. 22, 12-18.

Sano, F. J. Beta motion thresholds. Journal of Experimental Psychology, 1963, 66, 281-285.

SHe PARd, R. N., \& JudD, S. A. Perceptual illusion of rotation of three-dimensional objects. Science, 1976, 191, 952-954.

Uluman, S. The interpretation of visual motion. Cambridge, Mass: MIT Press, 1979.

Wertheimer, M. Experimentelle Studien über das Sehen von Bewegung. Zeitschrift für Psychologie, 1912, 61, 161-265.

(Manuscript received July 6, 1982; revision accepted for publication November 5, 1982.) 